\title{
A guide to bullous lesions of the skin
}

\author{
N. F. C. GANE \\ From the Department of Pathology, Godfrey Huggins School of Medicine, University of Rhodesia, Salisbury, \\ Rhodesia
}

SYNOPSIS A simple sequential chart for identifying bullous skin lesions is presented based on the site and size of the bulla and helpful changes in the bulla, epidermis, and dermis.

Bullous skin lesions are frequently a source of dismay to the beginner in histopathology since descriptions of the different lesions are scattered in textbooks among the various chapters on congenital, inflammatory, and toxic conditions. Sleuthing through these chapters does help to familiarize the beginner with these texts but the exercise is time-consuming and often discourages him from an intriguing subject. This chart seeks not to replace the perusal of textbooks but to simplify and even encourage this.

The chart (pp. 236-237) should be worked through from left to right, the initial basis of identification being the situation of the lesion in the skin.

\section{The Situation of the Lesion}

Only four situations are used: suprabasal, intraepidermal, subcorneal, and subepidermal. The main snare lies with the subepidermal group since the floor of the bulla may re-epithelialize rapidly and cause the apparent 'migration' of the bulla to a higher position. The pathologist must rely on the clinician to biopsy an early lesion in all bullous conditions.

Four descriptive terms are used for the size of a lesion. A lacuna $(\mathrm{L})$ is a small gap between normally adjacent structures. This may expand laterally to form a cleft (c) or circumferentially to form a roughly rounded bleb or vesicle $(v)$. The difference between a vesicle and its larger relative, the bulla (B), is one of size and is interpreted subjectively by different pathologists. The author regards bullae as unsightly but vesicles of a socially polite size.

Lymphangioma circumscriptum is appended to the subepidermal group since it is occasionally mistaken as a bullous lesion.

\section{Changes within the Lesion}

Acantholytic cells and inflammatory cells in the fluid of a bulla help to narrow the diagnostic Received for publication 10 January 1973. spectrum. Acantholysis is graded from + to +++ depending on its prominence.

\section{Adjacent Epidermal Changes}

Those listed are the presence of villi (hyperplastic accentuations of the normal dermal papillae), hyperkeratosis plus the occasional mention of parakeratosis ('para'), spongiosis, and acanthosis.

\section{Other Useful Features}

Only the more important features which help to clarify the diagnosis have been included. It is often impossible to distinguish incontinentia pigmenti and subcorneal pustular dermatosis from infections, and a warning of this is inserted here within brackets.

\section{Changes in the Dermis}

These may be extremely useful but in many conditions the only abnormality is a chronic inflammatory cell infiltrate (CICI).

\section{Affected Sites of the Body}

The distribution of lesions may differentiate conditions with similar histological appearances. The clinician often bases his diagnosis on this distribution but omits to mention it on the request form.

\section{Age}

Only those conditions with a definite predilection for certain periods of life are indicated.

\section{References}

Lever, W. F. (1967). Histopathology of the Skin, 4th ed. Lippincott, Philadelphia, Pitman, London.

Rook, A., Wilkinson, D. S., and Ebling, F. J. G. (1968). Textbook of Dermatology. Blackwell, Oxford. 


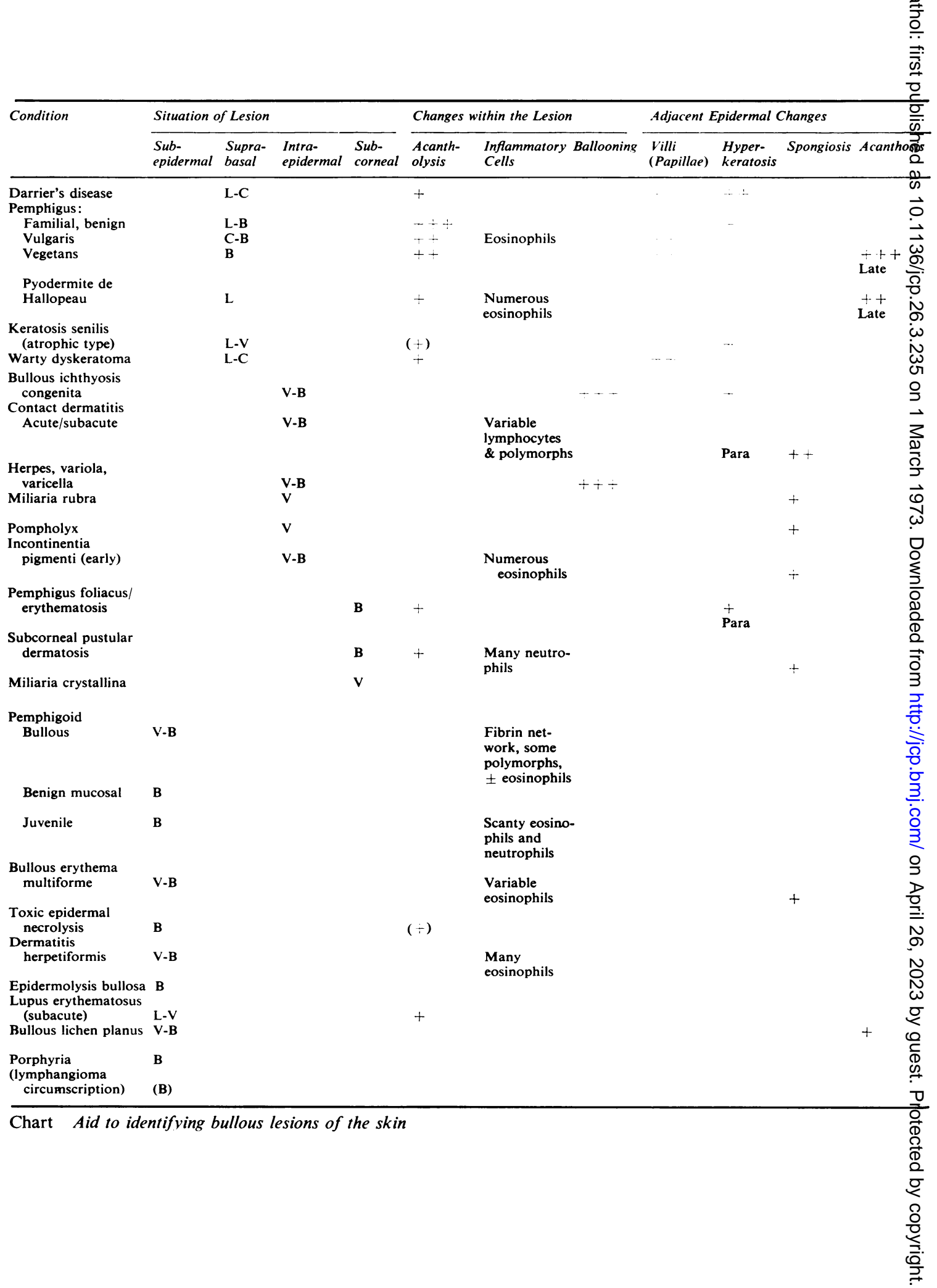


Corps ronds and grains, papillomatosis $\mathrm{CICI}$

Scanty corps and grains

Acantholytic cells degenerate

Late intraepithelial abscesses of eosinophils

Resemble pustules

Basal layer atypia

Central keratin plug

Pyknotic nuclei to cells

Reticular degeneration

Inclusion bodies, reticular degeneration Varying $\mathrm{CICI}$ vasculitis

Inflammation in epidermis around vesicles

Vesicles multiple and close together

(Confused with erythema toxicum neonatorum)

Bullae rupture early, occasional grains

(Confused with impetigo)

PAS-positive material in mouths of sweat glands

Initially multiple small vesicles

Variable epidermal necrosis

Degeneration of epidermis

Microabscesses in papillae

Basal cell vacuolation

Basal cell liquefaction

Saw-tooth epithelial downgrowths, basal cell degeneration

\section{CICI}

Mild inflammation

Eosinophils

CICI (may be prominent)

Perivascular CICI

Oedema and perivascular CICI

CICI

Mild CICI

Eosinophils

CICI and eosinophils

Scanty polymorphs around vessels

Mild oedema to severe vasculitis

Nil to marked inflammation, late fibrosis

CICI vasculitis

Nil to mild inflammation

CICI with eosinophils

Nil

CICI round appendages, collagen degeneration and oedema

Band-Like CICI, hugging epidermis

PAS-positive material and oedema of capillary walls (dilated superficial lymphatics)
Seborrhoeic areas, mucosae occasionally

Neck, axillae, groins

Skin and mouth

Flexures mainly

Exposed areas

Face, scalp, neck

Flexural surfaces

Infancy

Dependent on virus

Flexures, sweaty areas

Palms and soles

Limbs and flanks

Baby girls

Scalp, face, upper body

Abdomen, axillae, groins

Trunk

Limbs, groins, axillae

Elderly

Mucosae, conjunctivae, genitalia, skin

Lower trunk, buttocks, face

Under 5 yr

Extensor surfaces and mucosae

May involve whole body

20-50 yr

Extensor surfaces and buttocks

Occasionally children

Skin or mucosae

Face, chest, limbs

Infants

$\operatorname{Max} 30-40$

Skin and mucosae

Exposed areas

Chart Aid to identifying bullous lesions of the skin-continued

'Dyskaryotic or necrotic degeneration of acantholytic cells noted 